\title{
Applying Simulation Modelling in Quantifying Optimization Results
}

\author{
Ratko Stanković*, Diana Božić
}

\begin{abstract}
Improvements achieved by applying linear programming models in solving optimization problems in logistics cannot always be expressed by physically measurable values (dimensions), but in non-dimensional values. Therefore, it may be difficult to present the actual benefits of the improvements to the stake holders of the system being optimized. In this article, a possibility of applying simulation modelling in quantifying results of optimizing cross dock terminal gates allocation is outlined. Optimal solution is obtained on the linear programming model by using MS Excel spreadsheet optimizer, while the results are quantified on the simulation model, by using Rockwell Automation simulation software. Input data are collected from a freight forwarding company in Zagreb, specialized in groupage transport (Less Than Truckload - LTL).
\end{abstract}

Keywords: cross docking; gates allocation; optimization; quantifying; simulation

\section{INTRODUCTION}

Because of the growing competition on the global market and more complex customers' requirements, freight forwarding companies must constantly improve their operations and create competitive advantages in terms of timing, quality and cost. For a groupage transport (Less than Truck Load - LTL) specialized freight forwarding company, optimal cross dock gates assignment is one of the crucial factors in improving the performance of the cross docking operations [1]. Handling of freight in a cross dock terminal is labour intensive, and workers must quickly transfer freight during a short time period [2]. This is a typical optimization problem in logistics, which can be solved by methods of linear programming [3].

Input data are collected from a freight forwarding company in Zagreb, specialized in groupage transport. Optimal solution of the company cross dock receiving gates is obtained on the linear programming model, by using MS Excel spreadsheet optimizer Solver. Since the improvement comparing to the company empirical solution cannot be quantified in physically measurable values, it isn't suitable for presentation to the company stakeholders.

In order to quantify the improvement achieved by optimization in units of time, which is in this case the most important key performance indicator (KPI), the simulation model of inbound cross docking operations is designed, by using Rockwell Automation simulation software Arena.

Two simulation experiments, with the same input data are performed, first with the empirical solution the company normally use and the second with the optimized solution obtained from the linear programming model. The results of the experiments enable comparing throughput time (duration of cross docking operations) as the KPI which is required.

This way, the simulation modelling enables quantifying the improvement of cross docking operations in physically measurable values, in case when the linear programming model objective function is expressed as a non-dimensional value.

\section{CROSS DOCKING AND GROUPAGE TRANSPORT}

Generally, a cross dock terminal is a point in the supply chain where incoming shipments are transferred to outgoing delivery vehicles, with minimal internal processing and storage delay time. The main purpose is to enable economies in transportation by shipment consolidation but also a reduction of lead time and a decrease of inventory levels. In a cross dock terminal, shipments are unloaded from incoming trucks, moved across the terminal, sorted by outbound destinations and finally loaded onto outgoing trucks [2].

Groupage transport or Less-than-Truck-Load (LTL) is a type of road transport, based on consolidating (grouping together) shipments dispatched by several different shippers, for several different consignees, into the same truck load. The main benefit of consolidation is an optimal utilization of the vehicle payload, which enables reduction of transport costs. Besides the road network, the main infrastructure of groupage transport are cross dock terminals (XD terminals), operated by freight forwarders.

Groupage transport is an important segment of the freight forwarders services, therefore any improvement in cross docking performances have significant impact on their competitiveness [4].

In order to get relevant information and input data, analysis of the freight forwarding company cross docking operations, focussed to the receiving gates allocation, was performed in February 2021. Due to limitations of the software tools (academic versions) only a representative segment of the XD terminal is modelled, both by the linear programming model and by simulation model. However, the conclusions drawn from the results of the optimization process and from the optimization experiments may be considered relevant for the entire system.

\section{OPTIMIZING RECEIVING GATES ALLOCATON BY APPLYING LINEAR PROGRAMMING MODEL}

Optimization of the XD terminal gates assignment is actually a resource allocation problem, which can be mathematically depicted by a linear programing model, the 
resource allocation model. Optimal assignment of the XD terminal gates to the incoming trucks is related to the quantity of goods (number of pallets) for each destination zone and Manhattan distance between each receiving gate and the respective consolidation zone.

\subsection{Manhattan Distance}

As shown in Fig. 1, Manhattan Distance or Taxicab Distance is the distance between two points measured along axes at right angles. The name alludes to the grid layout of the streets of Manhattan, which causes the shortest path a car could take between two points in the city.

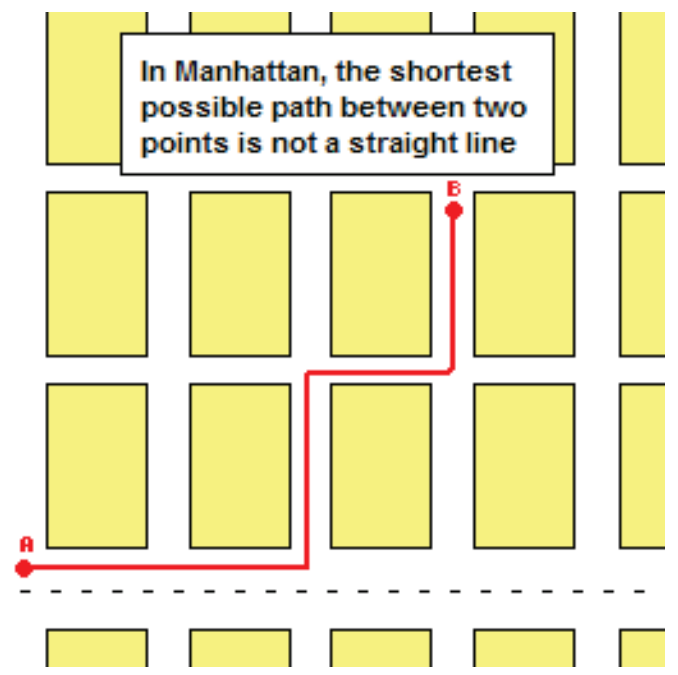

Figure 1 Manhattan Distance [5]

The reason for implementing Manhattan Distance instead of Euclidean Distance is because forklifts also follow similar paths within the XD terminal (instead of buildings there are pallet racks or rows). Also the actual distances in meters are not important, but ratios of distances between the gates and the consolidation zones, as shown in Fig. 2. The distances in the mathematical model are expressed in unit Manhattan Distances, where the distance between a gate and the closest consolidation zone $\left(d_{11}, d_{22}, d_{33}, d_{44}\right)$ equals 1 unit.

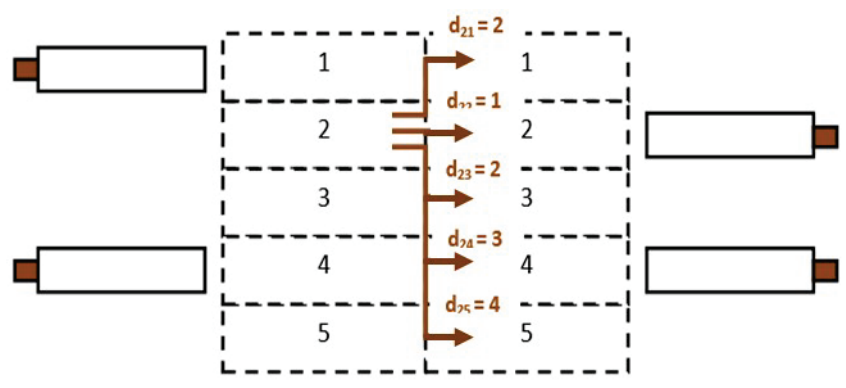

Figure 2 Unit Manhattan Distances in the XD terminal [6]

Manhattan Distance $d_{1}$ between two vectors $p=\left(p_{1}, p_{2}\right.$, $\left.\ldots, p_{n}\right)$ and $q=\left(q_{1}, q_{2}, \ldots, q_{n}\right)$ in $\mathrm{n}$-dimensional space is given by the Eq. (1).

$$
d_{1}(p, q)=\|p-q\|_{1}=\sum_{i=1}^{n}\left|p_{i}-q_{i}\right|
$$

\subsection{Mathematical Model}

A linear programming model is designed to mathematically describe the problem of optimizing the receiving gates allocation to incoming trucks, as follows.

\section{Objective function:}

- To minimize the quantity of goods moved and the distances passed by the forklifts (sum of products of quantities and respective distances), as given by the Eq. (2).

Constraints:

1) One receiving gate must be allocated to each inbound truck, as given by the inequity (3).

2) Each receiving gate can be allocated to only one or none inbound truck, as given by the inequity (4).

Input data:

- Number of inbound trucks;

- Quantity of goods (number of pallets) for each consolidation/destination zone, in each inbound truck;

- Total number of receiving gates;

- Total number of consolidation zones;

- Manhattan Distances from the receiving gates to the consolidation zones.

The mathematical model that encompasses all the elements of the problem is given by the following mathematical structure:

$$
\begin{aligned}
& \min F=\sum_{i=1}^{n} \sum_{j=1}^{m} \sum_{k=1}^{l} q_{k j} \cdot d_{i j} \cdot x_{i k} \\
& \sum_{i=1}^{n} x_{i k}=1 \quad \forall k=1, \ldots, l \\
& \sum_{k=1}^{l} x_{i k} \leq 1 \quad \forall k=1, \ldots, n \\
& x_{i k} \in\{0,1\} \quad \forall i=1, \ldots, n \\
& n \geq l
\end{aligned}
$$

Where:

$q_{k j}$ - number of pallets in inbound truck $k$ for consolidation zone $j$

$d_{i j}$ - Manhattan Distance between receiving gate $i$ and consolidation zone $j$

$x_{i k}$ - decision variable (binary): 1 - receiving gate $i$ allocated to incoming truck $k ; 0$ - receiving gate $i$ not allocated to incoming truck $k$

$n$ - total number of receiving gates

$m$ - total number of consolidation zones

$l$ - total number of trucks

Mathematical expression (5) defines the decision variable $x_{i k}$ as binary. Inequity (6) prevents the total number of inbound trucks to exceed the total number of receiving 
gates. This in fact wouldn't be an issue, since in such situation a queue is created.

\subsection{Empirical Solution of the Problem}

Empirical solution the company normally applies is based on a simple principle that the inbound truck that has the greatest number of pallets for a particular consolidation zone, gets the receiving gate closest to that zone. The truck with the second greatest number of pallets for a particular consolidation zone gets the next receiving gate closest to that zone, unless the gate is already allocated. Then it gets the second closest gate and so on. It is somewhat alike the Clarke \& Wright's Savings Algorithm (1964), although in the company they are not aware of that.

The value of the objective function, calculated with the empirical solution in MS Excel spreadsheet, is shown in Fig. 3.

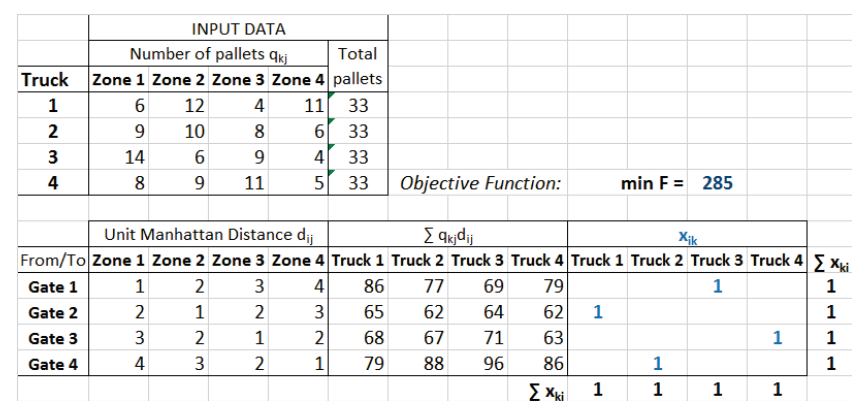

Figure 3 Empirical solution

In compliance to the a.m. empirical solving principle, Gate 1 is allocated to Truck 3, Gate 2 is allocated to Truck 1, Gate 3 is allocated to Truck 4 and Gate 4 is allocated to Truck 2 . This solution of the receiving gates allocation yields the objective function value of 285 (non-dimensional value).

\subsection{Optimal Solution of the Problem}

The optimal solution (Fig. 4) of the same problem is obtained by optimizing the mathematical model of the problem, using MS Excel spreadsheet optimizer Solver.

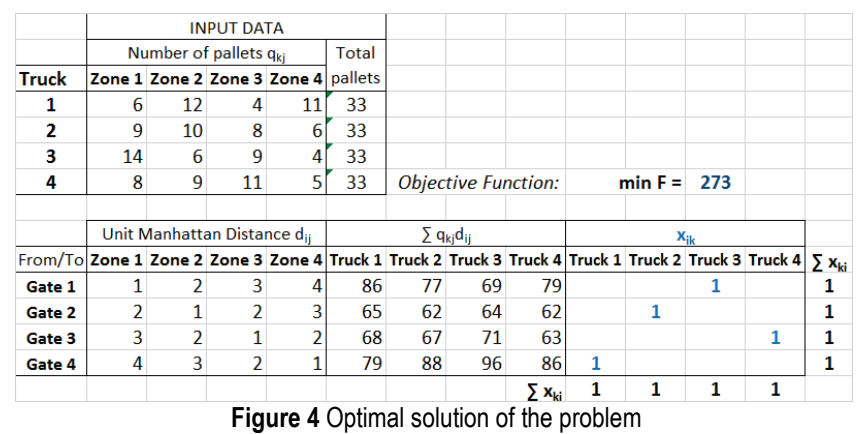

In the optimal solution, Gate 1 is allocated to Truck 3, Gate 2 is allocated to Truck 2, Gate 3 is allocated to Truck 4 and Gate 4 is allocated to Truck 1. This solution of the receiving gates allocation yields the objective function value of 273 (non-dimensional value).

\section{SIMULATION MODEL AND RUNNING SIMULATION EXPERIMENTS}

Since the values of the mathematical model objective function are non-dimensional values, while the improvement should be quantified in units of time, as the key performance indicator (KPI), the simulation model of inbound cross docking operations is designed, by using Arena simulation software. Arena is a discrete event simulation software developed by Rockwell Automation Inc. Discrete event simulation describes a process with a set of unique, specific events in time. It is designed for analysing the impact of changes involving significant redesigns associated with supply chain, manufacturing, logistics, distribution, warehousing and service systems.

The input data for the model are durations of the inbound cross docking operations, which are continuous stochastic variables. In order to determine the statistical distribution of the duration times, a representative sample is needed.

For this purpose, the inbound cross docking operations were scanned during February 2021 and 100 records of each movement were made. Due to limited resources, certain approximations have been introduced, but that is irrelevant for the main objective of this article.

Based on the empirical data, theoretical functions of probability distributions are determined, by using Arena Input Analyser software tool. The input data analysis resulted by selecting normal distribution of the cross docking operations duration times, as shown in Fig. 5.

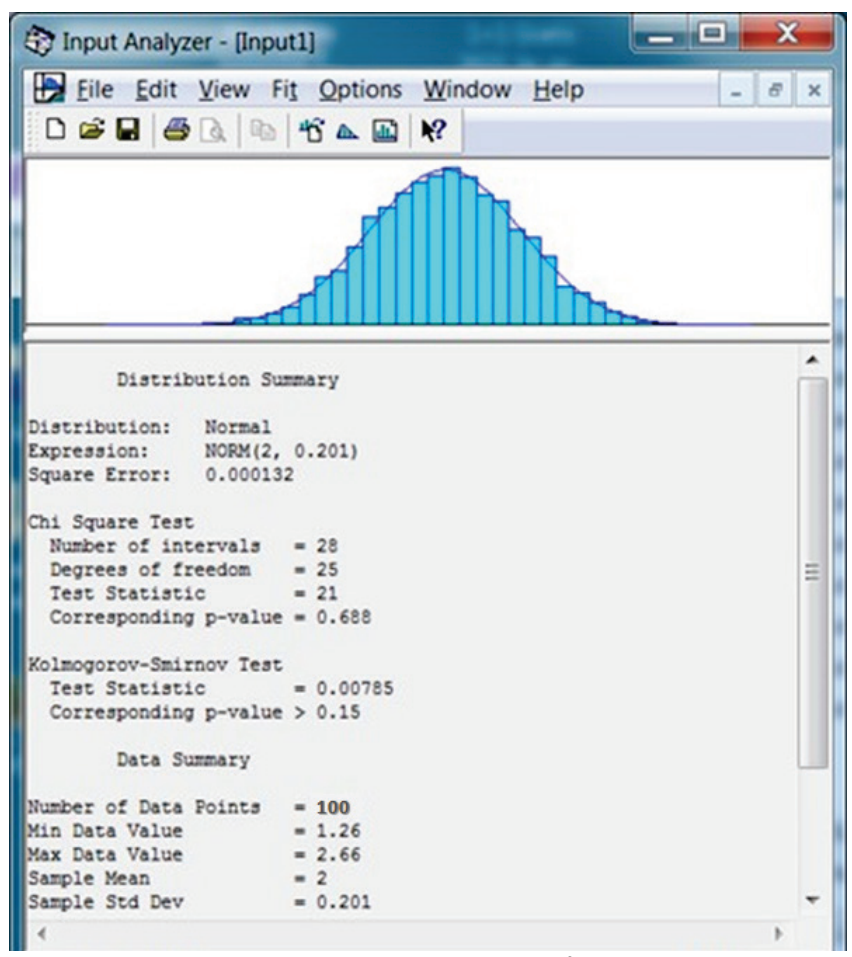

Figure 5 Theoretical probability distribution of the duration time

The distribution shown in Fig. 5 refers to movements from a receiving gate to the closest consolidation zone (e.g. Move from Gate 1 to Consolidation Zone 1) and is 
considered a unit time. Analogously to the Manhattan Distance calculation, durations of movements in other combinations Gate - Zone are listed in the Tab. 1.

Table 1 Duration times of the inbound cross docking operations

\begin{tabular}{|c|c|c|c|}
\hline $\begin{array}{c}\text { Operation } \\
\text { (Gate - Zone) }\end{array}$ & $\begin{array}{c}\text { Probability } \\
\text { Distribution }\end{array}$ & $\begin{array}{c}\text { Mean } \\
\text { (minute) }\end{array}$ & $\begin{array}{c}\text { Standard deviation } \\
\text { (minute) }\end{array}$ \\
\hline Move 1,1 & Normal & 2 & 0.201 \\
\hline Move 1,2 & Normal & 3 & 0.201 \\
\hline Move 1,3 & Normal & 4 & 0.201 \\
\hline Move 1,4 & Normal & 5 & 0.201 \\
\hline Move $i, j$ & Normal & $\ldots$ & 0.201 \\
\hline Move $\ldots$ & Normal & $\ldots$ & 0.201 \\
\hline Move $n, m$ & Normal & 2 & 0.201 \\
\hline
\end{tabular}

With reference to the principle of determining the cross docking operations duration times illustrated in the Tab. 1, the actual duration times generated in the simulation experiments are shown in the Fig. 6.

The key performance indicator (KPI) refers to the average time needed to move one pallet from the gate to the respective consolidation zone.

Two simulation experiments, with the same input data are performed, first with the empirical solution the company normally use and the second with the optimized solution obtained from the linear programming mathematical model.

\begin{tabular}{lcccr} 
Time per Entity & \multicolumn{5}{c}{} \\
VA Time Per Entity & & & & \\
& Average & Half Width & $\begin{array}{c}\text { Minimum } \\
\text { Value }\end{array}$ & $\begin{array}{r}\text { Maximum } \\
\text { Value }\end{array}$ \\
\hline Move 11 & 1.9809 & (Insufficient) & 1.6863 & 2.3889 \\
Move 12 & 3.0188 & (Insufficient) & 2.8838 & 3.2575 \\
Move 13 & 4.0850 & (Insufficient) & 3.7764 & 4.3115 \\
Move 14 & 5.0907 & (Insufficient) & 4.7589 & 5.3055 \\
Move 21 & 3.1603 & (Insufficient) & 2.8820 & 3.5047 \\
Move 22 & 1.9993 & (Insufficient) & 1.6983 & 2.2504 \\
Move 23 & 3.1324 & (Insufficient) & 2.8860 & 3.3447 \\
Move 24 & 3.9129 & (Insufficient) & 3.4525 & 4.1800 \\
Move 31 & 4.0321 & (Insufficient) & 3.7462 & 4.4033 \\
Move 32 & 3.1736 & (Insufficient) & 3.0628 & 3.3031 \\
Move 33 & 1.9427 & (Insufficient) & 1.5517 & 2.2864 \\
Move 34 & 2.9198 & (Insufficient) & 2.6234 & 3.1254 \\
Move 41 & 4.9925 & (Insufficient) & 4.5248 & 5.4973 \\
Move 42 & 2.9400 & (Insufficient) & 2.5340 & 3.2010 \\
Move 43 & 3.0656 & (Insufficient) & 2.9712 & 3.1975 \\
Move 44 & 2.0982 & (Insufficient) & 1.8300 & 2.3185
\end{tabular}

Figure 6 Duration times in the simulation experiments

\subsection{Simulation Experiment with the Empirical Solution}

Process flow of the simulation model with the empirical receiving gates allocation is shown in Fig. 7.

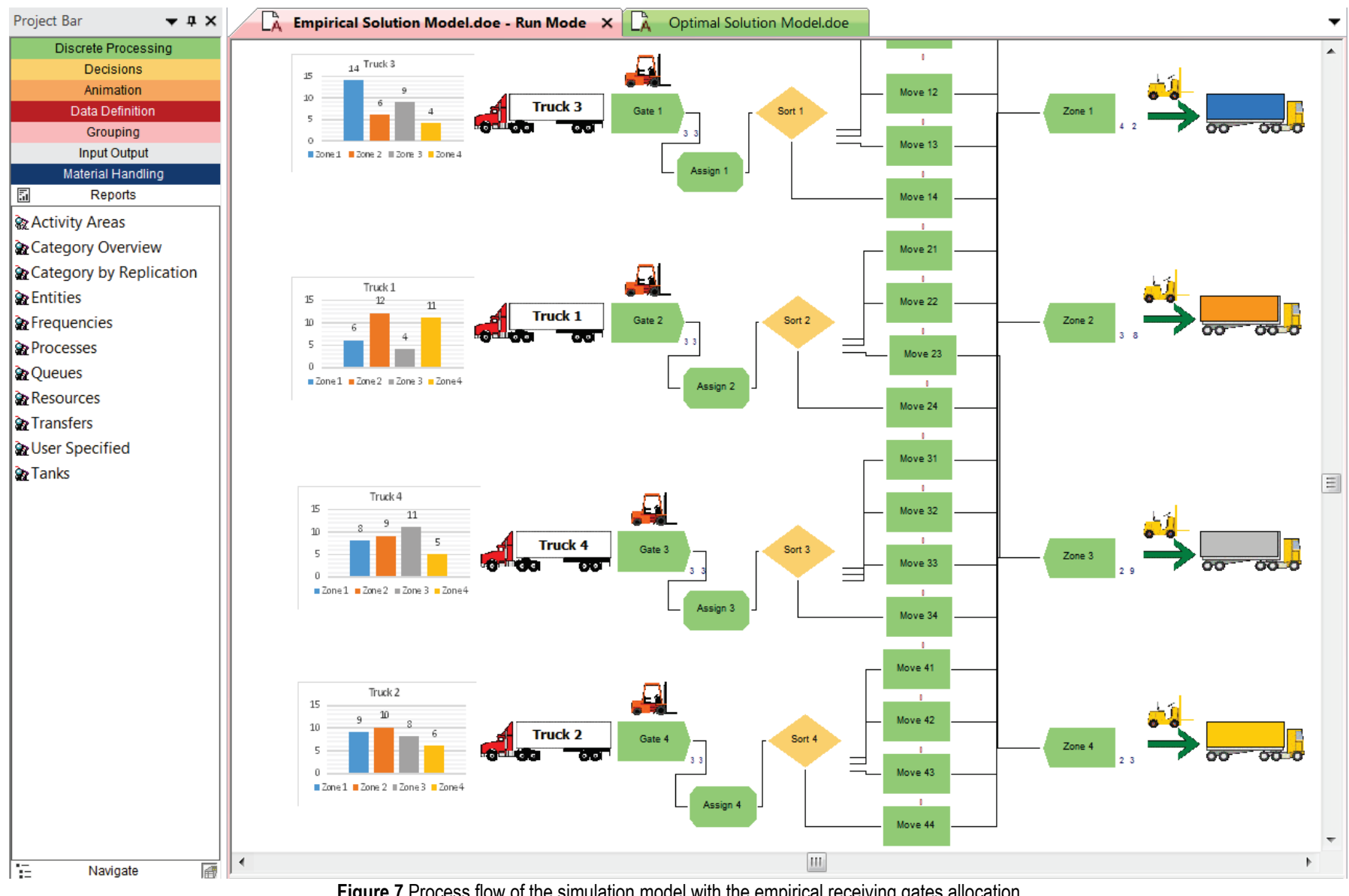

The value of the key performance indicator (KPI) obtained by the simulation experiment with the empirical receiving gates allocation is 3.30 minutes per pallet (the data generated in the Arena simulation reports).

\subsection{Simulation Experiment with the Optimal Solution}

Process flow of the simulation model with the optimal receiving gates allocation is shown in Fig. 8 . 
The value of the key performance indicator (KPI) obtained by the simulation experiment with the optimal receiving gates allocation is 3.16 minutes per pallet (the data generated in the Arena simulation reports).

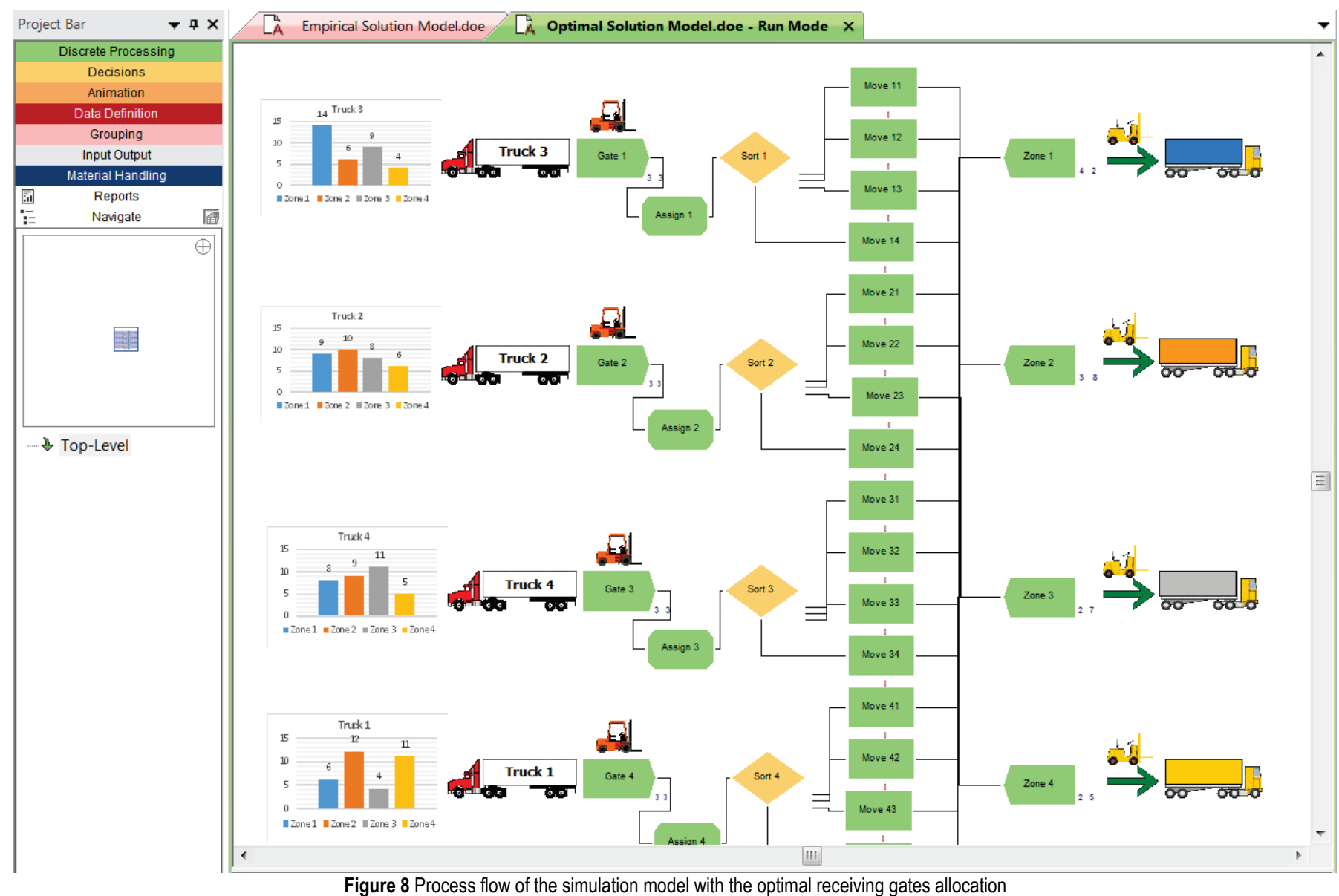

\section{DISCUSSION OF THE RESULTS}

The improvements in performances of the inbound cross docking operations, achieved by optimizing the receiving gates allocation, are listed in the Tab. 2.

Table 2 Improvements achieved by optimization

\begin{tabular}{|l|c|c|c|}
\hline \multicolumn{2}{|c|}{ OPTIMIZATION MODEL } & \multicolumn{2}{c|}{ SIMULATION MODEL } \\
\hline & $\begin{array}{c}\text { Objective function } \\
\text { value }\end{array}$ & Minutes per pallet & Total minutes \\
\hline Empirical & 285 & 3.30 & 436.06 \\
\hline Optimal & 273 & 3.16 & 417.07 \\
\hline Reduction & $\mathbf{4 . 2 \%}$ & $\mathbf{4 . 4 \%}$ & $\mathbf{1 8 . 9 9}$ \\
\hline
\end{tabular}

The improvement on the optimization model (linear programming mathematical model) is quantified as the difference between the value of the objective function in case of the empirical receiving gates allocation (285) and the value in case of the optimal gates allocation (273).

Since the values are non-dimensional, only the percentage reduction (4.2\%) may be referred as the improvement achieved, although it still cannot be expressed in any physical dimension (weight, time, distance or so).

The results of the simulation experiments enable comparing processing time (average duration of inbound cross docking operations) as the KPI required.
The KPI value in the experiment with the empirical receiving gates allocation is 3.30 minutes per pallet and the total time to process all pallets unloaded from all four inbound trucks (132 pallets) is 436.06 minutes.

The KPI value in the experiment with the optimal receiving gates allocation is 3.16 minutes per pallet and the total time to process all pallets unloaded from all four inbound trucks (132 pallets) is 417.07 minutes.

Therefore, the improvement is quantified in minutes, as the reduction of processing time achieved by optimization of the receiving gates allocation: $4.4 \%$ or 18.99 minutes for processing all 132 pallets.

Academic version of the Arena simulation software is limited to the maximum of 150 entities, which are pallets in this model. Because of this limitation, only four receiving gates could have been encompassed by the model. Each gate is allocated to one truck, therefore receives 33 pallets, which means a total of 132 pallets are generated out of four receiving gates. Each additional gate would generate 33 pallets more, which would exceed the limit of 150 entities.

\section{CONCLUSION}

Optimal receiving gates allocation enables minimizing the quantity of goods moved and the distances passed by the 
forklifts in the XD terminal, thus reduces working hours and the duration of the cross docking operations. Due to the simplicity of use, flexibility and fast problem solving, the optimization by linear programming mathematical model is suited for daily use in real situations, even when deviations in schedule of trucks arrivals occur.

However, the inability to quantify the improvement achieved by the optimization in physical dimensions (weight, time, distance, or so), may be a major disadvantage in presenting the actual benefits to the stakeholders of the system being optimized.

In order to quantify the benefits achieved by the optimization in units of time, as the required key performance indicator (KPI), the simulation model of inbound cross docking operations is designed, and two simulation experiments are performed. First with the empirical receiving gates allocation and the second with the optimized receiving gates allocation, obtained from the linear programming mathematical model.

This way, the simulation modelling enables not only quantifying benefits of the optimization in physically measurable values, but also verifying the mathematical model.

\section{REFERENCES}

[1] Goddefroy, N. \& Mellaerts, A. (2013). Optimizing the Dock Door Assignment Problem in Cross-Docking Warehouses. Master Thesis, Universiteit Gent, Faculteit Economie en Bedrijfskunde.

[2] Babić, D., Stanković, R., \& Bajor, I. (2020). Špediterski poslovi u logističkoj djelatnosti. Fakultet prometih znanosti, Zagreb. (in Croatian)

[3] Yu, V. F., Sharma, D., \& Murty, K. G. (2008). Door Allocations to Origins and Destinations at Less-than-Truckload Trucking terminals. Journal of Industrial and Systems Engineering, 2(1), 1-15.

[4] Stanković, R., Šafran, M., \& Božić, D. (2016). Guidelines for Improving Logistic Performances as Drivers of the Logistic Industry Development. Tehnički vjesnik - Technical Gazette, 23(5), 1497-1503. https://doi.org/10.17559/TV-20141016184435

[5] See https://www.joachimdespland.com\%2Fmammoth.html

[6] Đurić, N., Stanković, R., \& Pašagić Škrinjar, J. (2015). Optimizing the Cross-Dock Door Assignment by Applying Mathematical Model. International Scientific Conference ZIRP 2015, Zagreb.

\section{Authors' contacts:}

Ratko Stanković, PhD

Faculty of Transport and Traffic Sciences,

University of Zagreb,

ZUK Borongaj,

Borongajska cesta 83A, HR-10000 Zagreb, Croatia

ratko.stankovic@fpz.hr

Diana Božić, $\mathrm{PhD}$

Faculty of Transport and Traffic Sciences,

University of Zagreb,

ZUK Borongaj,

Borongajska cesta 83A, HR-10000 Zagreb, Croatia

diana.bozic@fpz.hr 